\title{
Editorial for the ISC'12 research paper sessions proceedings
}

\author{
Julian Kunkel · Thomas Ludwig • Hans Meuer
}

Published online: 23 May 2012

(C) Springer-Verlag 2012

The International Supercomputing Conference, founded in 1986 as the "Supercomputer Seminar", has been held annually for the last 25 years. Originally organized by Professor Hans Meuer, Professor of Computer Science at the University of Mannheim and former director of the computer centre, the Seminar brought together a group of 81 scientists and industrial partners who all shared an interest in high performance computing. Since then the annual conference has become a major international event within the HPC community, and accompanying its growth in size over the years, the conference has moved from Mannheim to Heidelberg and then to Dresden and finally to Hamburg. With 2,400 attendees and more than 160 exhibitors from over 50 countries expected to attend this year's conference, we are optimistic that this steady growth of interest will also turn ISC' 12 into a powerful and memorable event.

In 2007 we decided to strengthen the scientific part of the conference by presenting selected talks on relevant research results within the HPC field. These research paper sessions began as a separate day preceding the conference, where slides and accompanying papers were made available via the conference web site. The research paper sessions have since evolved into an integral part of the conference, and this year the scientific presentations are scheduled over a period of two days. The call for participation was issued in the winter of 2011, inviting researchers and developers to submit the latest results of their work as full research papers to the scientific sessions' program committee.

\footnotetext{
J. Kunkel $(\bowtie) \cdot$ T. Ludwig

University of Hamburg, Hamburg, Germany

e-mail: JulianKunkel@googlemail.com

H. Meuer

Prometeus, Waibstadt-Daisbach, Germany
}

A total of 32 papers were submitted from authors all over the world. In a peer-review process an international committee selected the best 16 papers for publication and for presentation in the research paper sessions. We are pleased to announce that a myriad of fascinating topics in HPC will be presented this year; the papers address the following issues in regards to the development of petascale supercomputers:

- Hardware and software co-design

- Performance analysis and optimization

- Scalable algorithms

- Energy efficiency

We believe that this selection is highly appealing across a number of specializations and that the presentations will foster inspiring discussions with the audience.

As in the years previous, two independent award committees have selected two papers considered to be of exceptional quality and worthy of special recognition.

The German-based Gauss Centre for Supercomputing sponsors the Gauss Award. This award is assigned to the most outstanding paper in the field of scalable supercomputing and goes to:

- Bluegene Team: Blue Gene/Q: By Co-Design

PRACE, the Partnership for Advanced Computing in Europe, awards a prize to the best scientific paper by a European student or scientist. This year's award is granted to:

- Y. Ineichen, A. Adelmann, C. Bekas, A. Curioni and P. Arbenz: A Fast \& Scalable Low Dimensional Solver for Charged Particle Dynamics in Large Particle Accelerators

The proceedings of the scientific sessions are published in a special issue of Computer Science-Research and Development. Thomas Ludwig (University of Hamburg and 
German Climate Computing Centre) and Julian Kunkel (German Climate Computing Centre) acted as guest editors.

We would like to express our gratitude to all our colleagues for submitting papers to the ISC scientific sessions, as well as to the members of the program committee for organizing this year's attractive program. We look forward to seeing you all in Hamburg and we wish you safe travels.

Hamburg, June 2012

Sincerely, Julian Kunkel Thomas Ludwig

Hans Meuer

\section{Program Committee Members}

- Pavan Balaji, Argonne National Lab, USA

- Reinhard Gerndt, University of Applied Sciences Braunschweig/Wolfenbüttel, Germany

- Galen Gisler, University of Oslo, Norway

- Lutz Gross, University of Queensland, Australia

- Georg Hager, University of Erlangen, Germany

- Jörg Henkel, Karlsruhe Institute of Technology, Germany

- Wolfgang Hiller, AWI for Polar \& Marine Research, Germany

- Sverre Jarp, CERN, Switzerland

- Julian Kunkel, German Climate Computing Centre, Germany

- Fang-Pang Lin, National Center for High-performance Computing (NCHC), Taiwan

- Thomas Ludwig, University of Hamburg and German Climate Computing Centre, Germany

- Federico Massaioli, CASPUR, Italy

- Simon Mcintosh-Smith, University of Bristol, United Kingdom

- Alexander Moskovsky, Russian Academy of Science, Russia

- Wolfgang E. Nagel, Technische Universität Dresden, Germany

- Kengo Nakajima, The University of Tokyo, Japan

- Stephan Olbrich, University of Hamburg, Germany

- Michael M. Resch, HLRS Stuttgart, Germany

- Yuichi Tsujita, Kinki University, Japan

- Zhonglei Wang, Karlsruhe Institute of Technology, Germany

\section{External Reviewers}

- Chen Chen

- Cihan Altinay

- Joel Fenwick

- Lin Gao

- Michael Kuhn

- Jan Treibig

- Gerhard Wellein

- Stephane Zuckerman

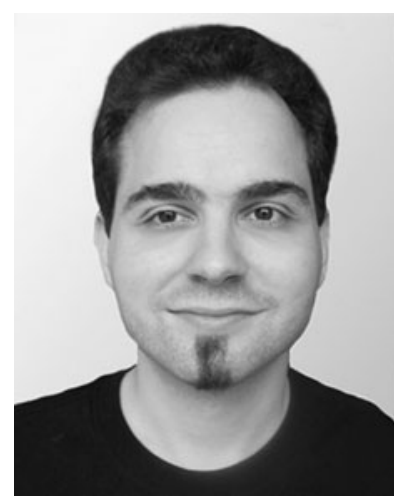

Julian Kunkel received his M.Sc. degree in computer science at the University of Heidelberg in 2007. Currently, he is employed at the German Climate Computing Centre. He invests his leisure time in his doctoral dissertation at the University of Hamburg. His interests cover high performance file systems and performance modeling of cluster systems.

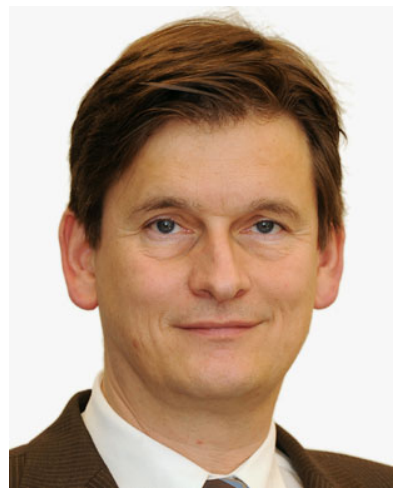

Thomas Ludwig received his doctoral degree and the German habilitation degree at the Technische Universität München, where he conducted research on HPC from 1988 to 2001. From 2001 to 2009 he had a chair for parallel computing at the Universität Heidelberg. Since 2009 he is the director of the German Climate Computing Centre (DKRZ) and professor at the Universität Hamburg. His research activity is in the fields of high volume data storage, energy efficiency, and performance analysis concepts and tools for parallel systems. At DKRZ Prof. Ludwig takes the responsibility for accomplishing its mission: to provide high performance computing platforms, sophisticated and high capacity data management, and superior service for premium climate science.

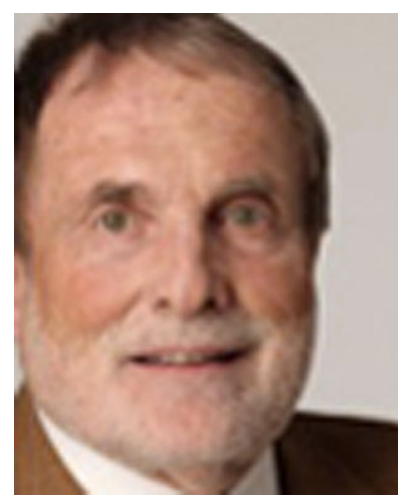

Hans Meuer ISC'12 General Chair, Prometeus \& University of Mannheim Prof. Dr. Hans Meuer is the Managing Director of Prometeus $\mathrm{GmbH}$ and the ISC General Chair. $\mathrm{He}$ is Professor Emeritus of Computer Science at the University of Mannheim, Department of Mathematics and Computer Science. In 1986, he became co-founder and organizer of the first Mannheim Supercomputer Conference, which has been held annually ever since. Hans Meuer has been the ISC General Chair from the very beginning. In 1993, Hans Meuer started the TOP500 initiative together with Erich Strohmaier and Jack Dongarra. Hans Meuer received his doctorate in mathematics in 1972 from RWTH Aachen University, Germany. He has been involved in data processing and computer science since 50 years. He served as specialist, project leader, group and department chief during his 11 years at the Research Center in Jülich, Germany, from 1962-1973. For the following 26 years, he was Director of the Computer Center at the University of Mannheim, Germany, and he has been Professor of Computer Science since 1974. Since 1998, he has been Managing Director of Prometeus $\mathrm{GmbH}$, the service company specialized in the field of High Performance Computing. 